\title{
Linear Projection Techniques in Damage Detection Under a Changing Environment
}

\section{Conference Paper}

\section{Author(s):}

Mozaffari, Salma (1); Döhler, Michael; Bernal, Dionisio P.; Liu, Yang

Publication date:

2014

\section{Permanent link:}

https://doi.org/10.3929/ethz-b-000487915

\section{Rights / license:}

In Copyright - Non-Commercial Use Permitted

Originally published in:

Conference Proceedings of the Society for Experimental Mechanics Series 7, https://doi.org/10.1007/978-1-4614-6585-0_30 


\title{
Linear Projection Techniques in Damage Detection under a Changing Environment
}

\author{
Salma Mozaffari Kojidi ${ }^{1}$, Michael Döhler ${ }^{1}$, Dionisio Bernal ${ }^{1}$, Yang Liu $^{2}$ \\ ${ }^{1}$ Department of Civil and Environmental Engineering, Center for Digital Signal Processing, \\ Northeastern University, 360 Huntington Ave., Boston MA 02115 \\ ${ }^{2}$ Harbin Institute of Technology, School of Transportation Science and Engineering, Harbin 150090, \\ China
}

\begin{abstract}
The merit of linear projections as a way to improve the resolution in damage detection under changing environmental conditions is examined. It is contended that if the data from the reference condition is balanced, in the sense that the number of feature vectors available for the various temperatures is similar, then projections, such as those in Principal Component Analysis and Factor Analysis, will not improve performance. Projections, however, help to control the false positive rate when the reference data set is not balanced. Analysis and simulation results suggest that previous claims on the merit of projection as a way to improve damage detection resolution under environmental variability may be too optimistic.
\end{abstract}

Keywords: Structural Health Monitoring, Damage Detection, Environmental Variability, Factor Analysis, Principal Component Analysis

\section{Introduction}

A difficulty in the use of SHM in civil engineering structures stems from the fact that any characterization used to describe the reference state is not a point in feature space but a hyper-volume (HV) whose boundaries depend on temperature, humidity, and other environmental variables. Making the reference state characterization conditional on a set of environmental variables reduces the dimension of the $\mathrm{HV}$ and this is the most effective way to treat environmental fluctuations, when feasible [1,2,3]. The possibility of performing detection without measurements of the environment, however, is of interest for cases where the formulation of an environmental model is deemed impractical.

If the environment is not compensated for, one expects that resolution will suffer because the reference state HV is large. A closer look, however, shows that not only the size, but also the shape of the HV enters the problem. Namely, if there are narrow dimensions in the HV, and the damage has a substantial projection in these directions, then reasonable resolution can still be realized. Since existence of narrow dimensions is a necessary condition for good performance, a question is how to check for their existence. A general answer is not trivial, because these dimensions can be curved in hyper-space, but for the simple case of constant directions, in feature space, they exist when there are small singular values in the covariance matrix of the data. Numerous claims have been made in the literature indicating that projection of the data in the subspace of the narrow dimensions followed by novelty detection in this subspace leads to improved performance. In this paper we examine the merit (or lack of) of these projections. 


\section{Basic Scheme}

The maximum likelihood estimate of the state of nature, given an observation $x$, is that for which the probability density of $x$ is highest. In damage detection it is often the case that only the probability density associated with the healthy state can be estimated and classification is reduced to deciding whether the point belongs to the reference state or not. This one class classification problems are carried out by selecting (in principle) some limit of the probability density bellow which the point is classified as "novel". In practice density is seldom computed explicitly and a surrogate is used instead. The Square Mahalanobis Distance (SMD) [4], which is proportional to the probability density when the distribution is multidimensional Gaussian, is perhaps most widely used. The SMD is defined as:

$$
d_{x}^{2}=(x-\mu)^{T} \Sigma_{x}^{-1}(x-\mu)
$$

where $\mu \epsilon R^{n}$ and $\Sigma_{x}$ are the mean vector and covariance matrix of the reference data, respectively. In this study we compare the performance of novelty detection based on the SMD in two instances: 1) when the distance is computed in the original space of the data and 2) when the distance is computed after the feature is projected to minimize the environmentally related variance. The SMD in the original space is given by eq.1 and in the projected space by eq.2

$$
d_{\varepsilon}^{2}=(\varepsilon-\bar{\varepsilon})^{T} \Sigma_{\varepsilon}^{-1}(\varepsilon-\bar{\varepsilon})
$$

where $\bar{\varepsilon}$ and $\Sigma_{\varepsilon}$ are the mean vector and covariance matrix of the projected reference data. We begin by summarizing the PCA and FA techniques.

\section{Principal Component Analysis}

Let $X=\left\{x_{1}, \ldots, x_{m}\right\}$, where $x_{i} \in R^{n}$, and $i=1, \ldots m$ be a data matrix where each column is a realization of a random process. An estimate of the covariance of the process is, by definition

$$
\Sigma_{x}=\frac{1}{m} \sum_{i=1}^{m}\left(x_{i}-\mu\right)\left(x_{i}-\mu\right)^{T}
$$

where $\mu$ is the mean vector. Recognizing that the covariance is symmetric, the singular value decomposition (SVD) gives

$$
\Sigma_{x}=U L U^{T}
$$

where $U$ is an orthogonal matrix with vectors $u_{1}, \ldots, u_{n}$ as its columns and $L$ is a diagonal matrix of singular values $l_{1}, \ldots, l_{n}$ $\left(l_{1}>\ldots>l_{n}\right)$. It often happens in practice that only a small numbers of singular values are large (relative to the rest) and the associated left side singular vectors are the principal components. In damage detection under environmental variability the projection is not in the principal components but in their complement, namely, the components that are associated with small singular values since these correspond to the smallest variability in the data. Formally, assuming that the last $(n-q)$ singular values are to be retained one has

$$
U=\left[\begin{array}{ll}
U_{1} & U_{2}
\end{array}\right] \quad L=\left[\begin{array}{cc}
L_{1} & 0 \\
0 & L_{2}
\end{array}\right]
$$

where $L_{1} \in R^{q \times q}, L_{2} \in R^{(n-q) \times(n-q)}, U_{1} \in R^{n \times q}, U_{2} \in R^{n \times(n-q)}$ and the projected feature vector is

$$
\varepsilon=U_{2}{ }^{T} x \quad\left(\varepsilon \in \mathbb{R}^{n-q}\right)
$$

Use of PCA in damage detection under environmental variability appears in [5].

\section{$\underline{\text { Factor Analysis }}$}

In factor analysis it is assumed that the data vector $x$ is generated by

$$
x=\mu+\Lambda \xi+\varepsilon
$$


where $\mu \epsilon R^{n}$ is the mean of the data, $\xi \epsilon R^{q}$ are the latent (unobserved) factors where $q<n$ and $\varepsilon \epsilon R^{n}$ is the residual or unique factor. It is evident by inspection that the decomposition in eq.7 is not unique. Two constraints that restrict the solution space are imposed: namely, one requires that: 1 ) the covariance of the factors is the identity and 2) the covariance of the residual $\Psi$ is diagonal. With these constraints the covariance of the model in eq.7 is

$$
\Sigma_{x}=\Lambda \Lambda^{T}+\Psi
$$

The most common approach to obtain $\Lambda$ and $\Psi$ from data is to use the Expectation Maximization algorithm [6]. In the literature the relevant equations appear as functions of the data but examination shows that the data itself is not relevant and that the equations reduce to

$$
\begin{gathered}
\Lambda=\Sigma_{\chi} \beta^{T}\left[I-\beta \Lambda+\beta \Sigma_{\chi} \beta^{T}\right]^{-1} \\
\Psi=\operatorname{diag}\left\{(I-\Lambda \beta) \Sigma_{\chi}\right\}
\end{gathered}
$$

where the diag operator sets all the off-diagonal elements of a matrix to zero, and $\beta$ is:

$$
\beta=\Lambda^{T}\left(\Psi+\Lambda \Lambda^{T}\right)^{-1}
$$

Eq.'s 9 to 11 are solved iteratively as follows: a) select initial values for $\Lambda$ and $\Psi$, b) compute $\beta$ from eq.11, c) compute new values of $\Lambda$ and $\Psi$ from eq.9 and eq.10, and repeat from (b) until convergence. The solution is unique for $\Psi$ and for the product $\Lambda \Lambda^{T}$ (which implies that the span of $\Lambda$ is uniquely determined). In the FA model the term $\Lambda \xi$ in eq.7 is assumed to contain most of the changes in the feature due to the environmental changes. The premise in FA is that when the system is damaged, the temperature effects no longer fit in the same span $\Lambda$ and this would be observable by inspecting the residual. To obtain the residuals, one computes the factors and uses eq.7. There are two main approaches to compute the factors: one is to use a weighted least squares solution, which is known as Bartlett's factor score

$$
\tilde{\xi}=\left(\Lambda^{T} \Psi^{-1} \Lambda\right)^{-1} \Lambda^{T} \Psi^{-1} x
$$

and the other, known as the Thomson's score, is

$$
\tilde{\xi}=\Lambda^{T}\left(\Psi+\Lambda \Lambda^{T}\right)^{-1} \chi
$$

Given the factors the residuals follow as

$$
\varepsilon=\mu+\Lambda \xi-x
$$

Use of FA for damage detection under environmental variability can be found in [7].

\section{Projection}

When projection is used, one trades the feature $x$ for the residual $\varepsilon$. Assume that the residual is linearly related to the feature by an invertible linear transformation, $P$, namely

$$
\varepsilon=P x
$$

In this case the SMD on $\varepsilon$ and $x$ are identical, namely:

$$
d_{\varepsilon}^{2}=(\varepsilon-\bar{\varepsilon})^{T} \Sigma_{\varepsilon}^{-1}(\varepsilon-\bar{\varepsilon})=(x-\mu)^{T} P^{T}\left(P \Sigma_{x} P^{T}\right)^{-1} P(x-\mu)=(x-\mu)^{T} \Sigma_{x}^{-1}(x-\mu)=d_{x}^{2}
$$

and it follows that if the SMD is used to decide on the novelty, a rank preserving transformation of the data vector is superfluous.

\section{Projection using PCA}

In PCA the projected vector is calculated using eq.6. Since $U_{2}$ is a wide matrix, PCA it is not a rank preserving transformation. To examine the relation between the SMD in the original and projected spaces we note that 


$$
\Sigma_{\varepsilon}=U_{2}^{T} \Sigma_{x} U_{2}=L_{2} \quad\left(\Sigma_{\varepsilon} \in \mathbb{R}^{(n-q) \times(n-q)}\right)
$$

so the $\mathrm{SMD}$ on $\varepsilon$ is:

$$
\begin{gathered}
d_{\varepsilon}^{2}=(\varepsilon-\bar{\varepsilon})^{T} \Sigma_{\varepsilon}^{-1}(\varepsilon-\bar{\varepsilon})=\left(U_{2}^{T} x-U_{2}^{T} \mu\right)^{T} L_{2}^{-1}\left(U_{2}^{T} x-U_{2}^{T} \mu\right) \\
=\left[U_{2}^{T}(x-\mu)\right]^{T} L_{2}^{-1} U_{2}^{T}(x-\mu)=(x-\mu)^{T} U_{2} L_{2}^{-1} U_{2}^{T}(x-\mu)
\end{gathered}
$$

Expressing the covariance in eq.1 in terms of its SVD, with a partition into significant and small singular values, (subscripts 1 and 2), one gets:

$$
\begin{gathered}
\mathrm{d}_{x}^{2}=(x-\mu)^{T} U_{1} L_{1}^{-1} U_{1}^{T}(x-\mu)+(x-\mu)^{T} U_{2} L_{2}^{-1} U_{2}^{T}(x-\mu) \\
\mathrm{d}_{x}^{2}=(x-\mu)^{T} U_{1} L_{1}^{-1} U_{1}^{T}(x-\mu)+\mathrm{d}_{\varepsilon}^{2}
\end{gathered}
$$

which shows that the SMD of the original data and of the projected data differ by the first term on the rhs of eq.21. Defining $y_{1}=U_{1}{ }^{T}(x-\mu)$ and $y_{2}=U_{2}{ }^{T}(x-\mu)$, which are the projections of $(x-\mu)$ to orthogonal subspaces $U_{1}$ and $U_{2}$, eq.21 becomes:

$$
\mathrm{d}_{x}^{2}=\mathrm{y}_{1}^{T} L_{1}^{-1} y_{1}+\mathrm{y}_{2}^{T} L_{2}^{-1} y_{2}
$$

where $y_{2}^{T} L_{2}^{-1} y_{2}=d_{\varepsilon}^{2}$. Consider performance under the null hypothesis. If there are an approximately equal number of data vectors for each of the temperature conditions, then the data matrix can be said to be "balanced", indicating that the mean vector $\mu$ is at the "center of gravity" of the data and the SMD in eq.22 can be expected to provide a good indicator of how likely any vector $x$ is. In contrast, if the data for a certain temperature distribution is poorly represented, vectors from this distribution will be "far from the mean", and the SMD will classify them (incorrectly) as novelty. Projection can help the Type I error in these cases but detection of damage with strong projections in the $\mathrm{U}_{1}$ direction is then difficult.

\section{Novelty Detection Using FA}

In FA the covariance of the factors is assumed to be the identity and the covariance of residuals is diagonal. To examine selfconsistency assume that the factors are computed using Thompson's score, in this case one finds that

$$
\begin{gathered}
\varepsilon=x-\Lambda \tilde{\xi}=x-\Lambda \Lambda^{T}\left(\Lambda \Lambda^{T}+\Psi\right)^{-1} x=\left(\left(\Lambda \Lambda^{T}+\Psi\right)\left(\Lambda \Lambda^{T}+\Psi\right)^{-1}-\Lambda \Lambda^{T}\left(\Lambda \Lambda^{T}+\Psi\right)^{-1}\right) x \\
=\left(\Lambda \Lambda^{T}+\Psi-\Lambda \Lambda^{T}\right)\left(\Lambda \Lambda^{T}+\Psi\right)^{-1} x=\Psi \Sigma_{x}^{-1} x
\end{gathered}
$$

therefore:

$$
\operatorname{cov}(\varepsilon)=\Psi \Sigma_{x}^{-1} \Psi
$$

so $\operatorname{cov}(\varepsilon) \neq \Psi$. Taking $P_{T h}=\Psi \Sigma_{x}^{-1}$, it follows that $\varepsilon=P_{T h} x$ and since $P_{T h}$ is full rank the SMD on $\varepsilon$ and $x$ are identical, making the computation of $\varepsilon$ superfluous for damage detection purposes.

Using Bartlett's estimation for the factors, the residual is found to be:

$$
\begin{gathered}
\varepsilon=x-\Lambda \xi=x-\Lambda\left(\Lambda^{T} \Psi^{-1} \Lambda\right)^{-1} \Lambda^{T} \Psi^{-1} x \\
=\left(I-\Lambda\left(\Lambda^{T} \Psi^{-1} \Lambda\right)^{-1} \Lambda^{T} \Psi^{-1}\right) x
\end{gathered}
$$

The question then is whether the term in the parenthesis in eq.25 is full rank, to make a determination we factor

$$
\Psi^{-1 / 2} \Lambda=Q R
$$

where $Q \epsilon R^{n \times q}$ has orthonormal columns $\left(Q^{T} Q=I\right)$ and $R \epsilon R^{q \times q}$ is an invertible matrix. Then the residual becomes: 


$$
\begin{gathered}
\varepsilon=\left(I-\Psi^{\frac{1}{2}} Q R\left(R^{T} Q^{T} Q R\right)^{-1} R^{T} Q^{T} \Psi^{-\frac{1}{2}}\right) x \\
=\left(\Psi^{\frac{1}{2}} \Psi^{-\frac{1}{2}}-\Psi^{\frac{1}{2}} Q Q^{T} \Psi^{-\frac{1}{2}}\right) x \\
=\Psi^{\frac{1}{2}}\left(I-Q Q^{T}\right) \Psi^{-\frac{1}{2}} x
\end{gathered}
$$

Define $P_{B}=\Psi^{\frac{1}{2}}\left(I-Q Q^{T}\right) \Psi^{-\frac{1}{2}}$, then $\varepsilon=P_{B} x$. In this case, the rank of $P_{B}$ is $(n-q)$, which is not full rank and one gathers that the SMD of the projection is not the same as that of the original data. We note that the covariance of $\varepsilon$ becomes

$$
\operatorname{cov}(\varepsilon)=P_{B} \Sigma_{x} P_{B}^{T}
$$

which, again, is not equal to $\Psi$. To have an invertible full rank covariance matrix the residual must be projected into a lower dimensional space. To do so let $Q_{2} \epsilon R^{n \times(n-q)}$ be orthonormal to $Q$ in eq.26, such that $\left[Q Q_{2}\right]$ is an orthogonal matrix of size $n \times n$. Then, $\left(I-Q Q^{T}\right)=Q_{2} Q_{2}{ }^{T}$. Defining a new environment-independent vector by normalizing $\varepsilon$ by $\Psi^{-1 / 2}$, projecting it into the subspace defined by $Q_{2}$, and using eq.27:

$$
\begin{gathered}
\tilde{\varepsilon}=Q_{2}^{T} \Psi^{-\frac{1}{2}} \varepsilon=Q_{2}^{T} \Psi^{-\frac{1}{2}}\left[\Psi^{\frac{1}{2}}\left(I-Q Q^{T}\right) \Psi^{-\frac{1}{2}} x\right] \\
=Q_{2}^{T}\left(Q_{2} Q_{2}^{T}\right) \Psi^{-\frac{1}{2}} x=Q_{2}^{T} \Psi^{-\frac{1}{2}} x
\end{gathered}
$$

where $\tilde{\varepsilon} \epsilon R^{n-q}$, and its covariance in the reference condition is

$$
\Sigma_{\tilde{\varepsilon}}=Q_{2}^{T} \Psi^{-\frac{1}{2}} \Sigma_{x} \Psi^{-\frac{1}{2}} Q_{2} \quad \Sigma_{\tilde{\varepsilon}} \in \mathbb{R}^{(n-q) \times(n-q)}
$$

where the term $\Psi^{-\frac{1}{2}} \Sigma_{\mathrm{x}} \Psi^{-\frac{1}{2}}$ can be simplified using eq.26 and eq.8:

$$
\begin{aligned}
& \Psi^{-\frac{1}{2}} \Sigma_{x} \Psi^{-\frac{1}{2}}=\Psi^{-\frac{1}{2}}\left(\Lambda \Lambda^{T}+\Psi\right) \Psi^{-\frac{1}{2}} \\
& =\Psi^{-\frac{1}{2}} \Lambda \Lambda^{T} \Psi^{-\frac{1}{2}}+I=Q R R^{T} Q^{T}+I
\end{aligned}
$$

Substituting eq.31 in eq.30 and recalling that $Q_{2}{ }^{T} Q=0$ (or $Q^{T} Q_{2}=0$ ), yields:

$$
\Sigma_{\tilde{\varepsilon}}=Q_{2}^{T}\left(Q R R^{T} Q^{T}+I\right) Q_{2}=0+Q_{2}^{T} I Q_{2}=I
$$

This covariance is full rank in the space of $\tilde{\varepsilon}$. Thus, the SMD on $\tilde{\varepsilon}$ to the reference data set after projection is defined as

$$
\mathrm{d}_{\tilde{\varepsilon}}^{2}=(\tilde{\varepsilon}-\overline{\tilde{\varepsilon}})^{T}(\tilde{\varepsilon}-\overline{\tilde{\varepsilon}})
$$

where $\overline{\tilde{\varepsilon}}=Q_{2}^{T} \Psi^{-1 / 2} \mu$. To inspect how the Mahalanobis of this projection compares to that in the original space we note that

$$
\begin{gathered}
\mathrm{d}_{x}^{2}=(x-\mu)^{T} \Sigma_{x}{ }^{-1}(x-\mu) \\
=(x-\mu)^{T} \Psi^{-\frac{1}{2}}\left(\Psi^{-\frac{1}{2}} \Sigma_{x} \Psi^{-\frac{1}{2}}\right)^{-1} \Psi^{-\frac{1}{2}}(x-\mu) \\
=(x-\mu)^{T} \Psi^{-\frac{1}{2}}\left(Q R R^{T} Q^{T}+I\right)^{-1} \Psi^{-\frac{1}{2}}(x-\mu)
\end{gathered}
$$




$$
\begin{gathered}
=(x-\mu)^{T} \Psi^{-\frac{1}{2}}\left(\left[\begin{array}{ll}
Q & Q_{2}
\end{array}\right]\left[\begin{array}{cc}
R R^{T}+I & 0 \\
0 & I
\end{array}\right]\left[\begin{array}{l}
Q^{T} \\
Q_{2}^{T}
\end{array}\right]\right)^{-1} \Psi^{-\frac{1}{2}}(x-\mu) \\
=(x-\mu)^{T} \Psi^{-\frac{1}{2}} Q\left(R R^{T}+I\right)^{-1} Q^{T} \Psi^{-\frac{1}{2}}(x-\mu)+(x-\mu)^{T} \Psi^{-\frac{1}{2}} Q_{2} Q_{2}^{T} \Psi^{-\frac{1}{2}}(x-\mu) \\
=(x-\mu)^{T} \Psi^{-\frac{1}{2}} Q\left(R R^{T}+I\right)^{-1} Q^{T} \Psi^{-\frac{1}{2}}(x-\mu)+(\tilde{\varepsilon}-\overline{\tilde{\varepsilon}})^{T}(\tilde{\varepsilon}-\overline{\tilde{\varepsilon}}) \\
=(x-\mu)^{T} \Psi^{-\frac{1}{2}} Q\left(R R^{T}+I\right)^{-1} Q^{T} \Psi^{-\frac{1}{2}}(x-\mu)+\mathrm{d}_{\tilde{\varepsilon}}^{2}
\end{gathered}
$$

which shows that the SMD of the original data and of the projected data differ by the first term on the rhs of eq.34. All one can say by looking at eq.34 is that the first term in eq.34 is negligible compared to the second when $\left(R R^{T}+I\right)>>I$, since then $\left(R R^{T}+I\right)^{-1}<<I$ and this appears to be usually the case.

\section{Summary}

The basic observations from the analytical examination are:

1) If the data matrix is balanced, no advantage is expected from projection (in either PCA or FA).

2) If the data matrix is unbalanced, projection can improve the Type I error rate, but this may lead to some degradation in the Type II error performance (both in PCA or FA).

3) When using FA, the factors need to be computed with Bartlett's score, otherwise the projection is rank preserving and thus superfluous for a Mahalanobis distance computation.

\section{Simulation Example}

This example is set up to validate the analytical observations. Consider a mass-spring system with 8 equal masses and initial stiffness $k_{0}$ as shown in figure 1.

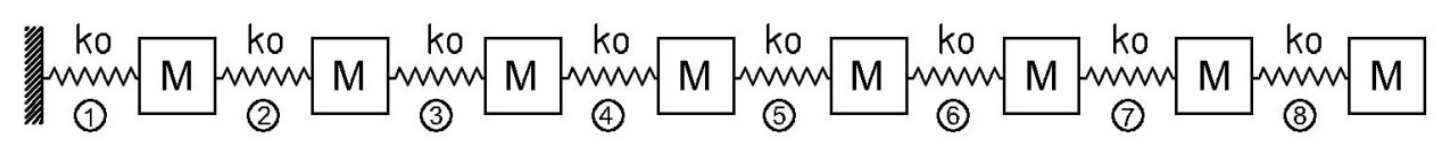

Fig.1 mass-spring system

The spring stiffness is assumed to be a function of temperature as:

$$
k=k_{0}\left(1+\frac{0.005}{20^{3}} T^{3}\right)
$$

where $T$ is the temperature in Celsius and $k_{0}$ is the stiffness at $T=0^{\circ} \mathrm{C}$. The temperature is assumed to have a yearly seasonal fluctuation that is harmonic plus a random component as shown in figure 2. The temperature in each spring is taken as the value from the ambient temperature plus an additional increment taken from a Gaussian distribution with zero mean and $0.5^{\circ} \mathrm{C}$ standard deviation. The feature vector consists of the first three frequencies, which, in the simulations are contaminated with white noise with a standard deviation of $0.2 \%$ of the mean of the frequency. Damage is simulated as a $10 \%$ reduction in each one of the springs (one at a time). 


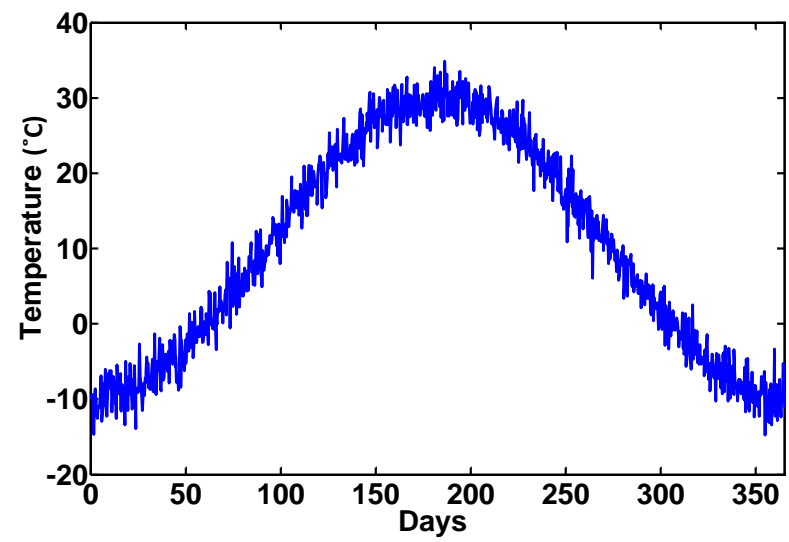

Fig.2 yearly temperature variation

The first year is used to formulate the reference model, a second year is used for validation and in the third year damage is introduced. We consider two reference state models, namely, one where the data matrix is balanced, obtained by sampling three times a day, and a second that is not balanced, obtained by sampling three times a day when the ambient temperature is below zero and once a day when it's above. In both cases the second year is used for validation and in the third damage is considered. Sampling in the second and the third year is three times per day. Results are presented in Table 1 for a balanced reference and in Table 2 for the unbalanced case. The Type I error is the number of false positives, the Power of the Test (POT) is the percent of the times that damage is identified, when it is present, and $p$ is the dimension of the projection space.

Table 1 Type I error and Power of the Test - balanced reference data

\begin{tabular}{|c|c|c|c|c|c|c|c|c|c|}
\hline & \multirow[b]{2}{*}{ Type I error (\%) } & \multicolumn{8}{|c|}{$\begin{array}{c}\text { Power of the test (\%) } \\
\text { (10 \% damage on Springs \#1-8 one at a time) }\end{array}$} \\
\hline & & $\# 1$ & $\# 2$ & $\# 3$ & $\# 4$ & \#5 & $\# 6$ & \#7 & \#8 \\
\hline No projection & 4 & 84 & 98 & 94 & 98 & 53 & 97 & 98 & 78 \\
\hline Projection (PCA, $p=1)$ & 5 & 41 & 99 & 91 & 15 & 8 & 12 & 87 & 48 \\
\hline Projection (PCA, $p=2$ ) & 6 & 45 & 99 & 93 & 92 & 40 & 98 & 80 & 57 \\
\hline
\end{tabular}

Table 2 Type I error and Power of the Test - unbalanced reference data

\begin{tabular}{|c|c|c|c|c|c|c|c|c|c|}
\cline { 3 - 12 } \multicolumn{1}{c|}{} & \multicolumn{9}{|c|}{ Power of the Test (\%) } \\
\cline { 2 - 12 } \multicolumn{1}{c|}{} & Type I error (\%) & $\mathbf{4 1}$ & $\mathbf{\# 2}$ & $\mathbf{\# 3}$ & $\mathbf{\# 4}$ & $\mathbf{\# 5}$ & $\mathbf{\# 6}$ & $\mathbf{\# 7}$ & $\mathbf{\# 8}$ \\
\hline No Projection & 14 & - & - & - & - & - & - & - & - \\
\hline Projection (PCA, p=1) & 8 & 74 & 98 & 93 & 40 & 16 & 11 & 65 & 38 \\
\hline Projection (PCA, p=2) & 6 & 79 & 99 & 96 & 90 & 51 & 98 & 61 & 46 \\
\hline
\end{tabular}

As anticipated, the Type I error is essentially the same whether one projects the data or not in the balanced data case and performance in the damaged state is superior when operating with the original data. The large improvement in the Type II error (in some cases) when $p$ goes from 1 to 2 is due to the fact that in this case the feature vector is only of dimension 3. Results for the case where the data is not balanced show that the Type I error without projection is unacceptable. In this case we do not report the POT since it would be misleading. Results for FA are not presented but proved analogous to the ones shown for the PCA projections. 


\section{Concluding Remarks}

The analysis presented suggests that projections do not improve performance if the data for the reference condition is balanced. The analyses and results do not support the claims that have been made in the literature about the gains in resolution attained by projection when the features vary with environmental conditions. The essential point is that feasibility depends on whether there are narrow dimensions, not on whether one projects the data. Indeed, it is contended that if the reference data is balanced, so the mean and the covariance are representative, then projection leads to a deterioration of the detector performance.

With regards to damage detection in real structures subjected to environmental changes it is essential to recognize that the problem becomes increasingly difficult as the size of the structure increases. This is so because the environmental effects act on the complete structure while damage is local. As the size increases, therefore, the importance of changes due to damage compared to changes due to environmental fluctuations decreases.

\section{Acknowledgement}

This research was supported by grant 1000391 NSF under the Hazard Mitigation and Structural Engineering Program Grant 1000391. This support is gratefully acknowledged.

\section{References}

[1] Worden, K., Sohn, H., \& Farrar, C. R. Novelty detection in a changing environment: regression and interpolation approaches. Journal of sound and vibration, 258(4), 741-761, 2002.

[2] Peeters, B. \& De Roeck, G. One year monitoring of the Z24 bridge: environmental influences versus damage effects. In Proc. IMAC-XVIII, San Antonio, TX, pp. 1570-1576, 2000.

[3] Sohn, H., Farrar, C. R., Hunter, N. F., \& Worden, K. Structural health monitoring using statistical pattern recognition techniques. Journal of dynamic systems, measurement, and control, 123, 706, 2001.

[4] Hotelling, H. Multivariate quality control illustrated by the air testing on samples of bombsights. Techniques of Statistical Analysis, 111-184, 1947.

[5] Yan, A. -M., Kerschen, G., De Boe, P., \& Golinval, J. -C. Structural damage diagnosis under varying environmental conditions-Part I: A linear analysis. Mechanical Systems and Signal Processing 19, 847-864, 2005.

[6] Rubin, D., \& Thayer, D. EM algorithms for ML factor analysis. Phcycometrika, 69-76, 1982.

[7] Kullaa, J. Is temperature measurement essential in structural health monitoring. In Proceedings of the 4th International Workshop on Structural Health Monitoring (pp. 717-724), 2003. 